\title{
MAGNETARS AND PULSARS: A MISSING LINK
}

\author{
Bing Zhang \\ Department of Astronomy $\&$ Astrophysics \\ Pennsylvania State University \\ bzhang@astro.psu.edu
}

\begin{abstract}
There is growing evidence that soft gamma-ray repeaters (SGRs) and anomalous X-ray pulsars (AXPs) are isolated neutron stars with superstrong magnetic fields, i.e., magnetars, marking them a distinguished species from the conventional species of spindown-powered isolated neutron stars, i.e., radio pulsars. The current arguments in favor of the magnetar interpretation of SGR/AXP phenomenology will be outlined, and the two energy sources in magnetars, i.e. a magnetic dissipation energy and a spindown energy, will be reviewed. I will then discuss a missing link between magnetars and pulsars, i.e., lack of the observational evidence of the spindown-powered behaviors in known magnetars. Some recent theoretical efforts in studying such behaviors will be reviewed along with some predictions testable in the near future.
\end{abstract}

Keywords: pulsars, magnetars, neutron stars, magnetic fields

\section{Soft gamma-ray repeaters and anomalous $\mathrm{X}$-ray pulsars as magnetars}

For a long time, radio pulsars have been regarded as the only manifestation of isolated neutron stars ${ }^{1}$. Recent observational developments indicate that isolated neutron stars also manifest themselves as other species (Pavlov et al. 2002, for a review), among which soft gamma-ray repeaters (SGRs) and anomalous X-ray repeaters (AXPs) have attracted growing attention in the neutron star community. These two types of objects originate, respectively, from the anomalous species of two distinct classes of phenomenon, i.e., gamma-ray bursts and accreting X-ray pulsars, but share many common features. Recently, two observational facts finally connect a bridge between SGRs and AXPs. First, after being quiescent for more than twenty years, SGR 0526-66 is found to have a steep non-thermal spectrum in the quiescent state which is similar to 
the non-bursting AXPs (Kulkarni et al. 2003). Second, soft, repeating bursts were recently detected from two AXPs, 1E 1048-5937 (Gavriil, Kaspi \& Woods 2002) and 1E 2259+586 (Kaspi \& Gavriil 2002). These suggest that SGRs/AXPs belong to a unified class of objects.

In the literature, there exist essentially four types of models to interpret SGR/AXP phenomenology. These are, according to the sequence of popularity, the magnetar model (Duncan \& Thompson 1992; Paczyński 1992; Thompson \& Duncan 1995, 1996; Thompson, Lyutikov \& Kulkarni 2002), the accretion model involving fossil disks (Chatterjee et al. 2000; Alpar 2001; Masden et al. 2001), the models involving strange quark stars (Alcock et al. 1986; Cheng \& Dai 1998; Zhang, Xu \& Qiao 2000; Usov 2001), and the models involving magnetic white dwarfs (Paczyński 1990; Usov 1993). It is fair to say that at the current stage none of the models can interpret all SGR/AXP observations satisfactorily. Nonetheless, the magnetar model has its merit to interpret most observations under one single hypothesis, i.e., SGRs/AXPs are neutron stars with superstrong magnetic fields ( $\sim 10^{14}-10^{15} \mathrm{G}$ at the surface). Other models either have troubles to interpret some observations (e.g. the accretion model fails to account for the super-Eddington SGR bursts) or have to introduce additional assumptions to account for data (e.g. Zhang 2002a for a review). Below I will list the solid observational facts of SGR/AXPs and confront them with the magnetar model.

1. Timing properties. Known SGRs/AXPs exclusively have long periods $[P \sim(5-12) \mathrm{s}]$ and large spindown rates $\left[\dot{P} \sim 5 \times\left(10^{-13}-10^{-10}\right)\right.$ $\mathrm{s} / \mathrm{s}]$. Assuming magnetic braking, this directly refers to a superstrong surface magnetic fields $\left[B_{s} \sim\left(10^{14}-10^{15}\right) \mathrm{G}\right.$ if these objects are neutron stars. Irregular spindown may be a common feature of these objects, and is not necessarily related to the bursting behavior. This could be accomendated in a magnetar model with twisted magnetosphere (Thompson et al. 2002).

2. Quiescent emission properties. SGRs/AXPs all display a steady luminous X-ray emission with $L_{x} \sim\left(10^{35}-10^{36}\right) \mathrm{ergs} / \mathrm{s}$, which could be explained in terms of magnetic dissipation (magnetic field decay, Thompson \& Duncan 1996; or magnetic enhanced cooling, Heyl \& Hernquist 1998; or untwisting of a global current-carrying magnetosphere, Thompson et al. 2002). Optical/IR counterparts have been detected from three AXPs (4U 0142+61, 1E 2259+586, and 1E 1048.15937), but no promising interpretation within the magnetar model is proposed. No gamma-ray and radio emission has been firmly detected from the SGRs/AXPs.

3. Burst properties. SGR bursts are soft and repeating, with luminosity ranging from $10^{38} \mathrm{ergs} / \mathrm{s}$ all the way up to $\sim 10^{45} \mathrm{ergs} / \mathrm{s}$ 
(usually super-Eddington, and two most luminous bursts, namely giant flares, have been detected from SGR 0526-66 on March 5, 1979; and from SGR 1900+14 on August 27, 1998). A strength of the magnetar model is that it can interpret the bursting phenomenology successfully in terms of the magnetic cataclysmic dissipation events in superstrong magnetic fields. Super-Eddington bursts are natural in strong fields in which the Thomson cross section is suppressed.

4. Environmental effects. Most SGRs/AXPs are located close to supernova remnants (SNRs) in projection. Solid associations with the SNRs are yet firmly established. Real associations are consistent with the magnetar theory which predicts that these objects are young neutron stars, but the SNR ages are not fully consistent with the spindown age of these objects. Assuming associations, SGRs have larger proper motions than AXPs. That one AXP with SNR association, 1E $2259+586$, recently displayed hundreds of repeating bursts make the issue more complicated. The claim that SGRs/AXPs are born in dense environments (Marsden et al. 2001) is not confirmed (Gaensler et al. 2001).

5. Cyclotron features. Cyclotron features have been detected in SGR outbursts (Ibrahim et al. 2002), which is consistent with the magnetar model if the features are of proton-origin, but refers to a much lower magnetic field if the features are of electron-origin.

In summary, though not fully unquestionable, the magnetar model is successful in many respects in interpreting the data. However, there is hitherto no definite proof that SGRs/AXPs are isolated neutron stars powered by superstrong magnetic fields. I believe that the key to prove the magnetar interpretation would be looking for a missing link between magnetars and normal pulsars, which I will lay out in the next section.

\section{Two energy sources in magnetars, and a missing link between magnetars and pulsars}

If SGRs/AXPs are magnetars, there should be two independent energy sources in these objects, i.e., the magnetic energy and the spin energy of a neutron star. Assuming a dipole geometry, the total magnetic energy in a magnetar magnetosphere is $E_{B} \simeq(1 / 12) B_{p}^{2} R^{3}$. Taking

$B_{p}=6.4 \times 10^{19} \mathrm{G} \sqrt{P \dot{P}}$, and $R=10^{6} \mathrm{~cm} R_{6}$, the magnetic energy can be estimated

$$
E_{B}=1.7 \times 10^{46} \operatorname{ergs}(P / 5 \mathrm{~s}) \dot{P}_{-11} R_{6}^{3},
$$

where $\dot{P}_{-11}=\dot{P} /\left(10^{-11}\right)$. The rotation energy of the magnetar is

$$
E_{R}=(1 / 2) I \Omega^{2}=7.9 \times 10^{44} \operatorname{erg} I_{45}(P / 5 \mathrm{~s})^{-2},
$$


where $I=10^{45} \mathrm{~g} \mathrm{~cm}^{3} I_{45}$ is the typical momentum of inertia of the magnetar. The critical line in the $P-\dot{P}$ diagram for the magnetic energy domination is

$$
\dot{P}_{-11}>5.8 P^{-3} I_{45} R_{6}^{-3} \text {. }
$$

In reality, what is more relevant is to compare the energy release rate of the magnetic energy and the spin energy. The former could be in principle written $L_{B}=d E_{B} / d t=-(1 / 6)\left(d B_{p} / d t\right) B_{p} R^{3}$. Theoretically, $d B_{p} / d t$ is rather uncertain. It is more straightforward to take $L_{B} \sim$ $10^{35}-10^{36} \mathrm{erg} \mathrm{s}^{-1}$ directly from the observations, e.g.

$$
L_{B}=10^{35} \operatorname{erg~s}^{-1} L_{B, 35}(B),
$$

where $L_{B, 35}(B)$ is an unknown function of $B$, but may be insensitive to $B$ when $B_{p} \sim 10^{14}-10^{15} \mathrm{G}$. The spindown luminosity is

$$
L_{s d}=-I \Omega \dot{\Omega}=4 \pi^{2} I P^{-3} \dot{P}=3.2 \times 10^{33} \mathrm{erg} \mathrm{s}^{-1} I_{45}(P / 5 \mathrm{~s})^{-3} \dot{P}_{-11} .
$$

Let $L_{B}>L_{s d}$, the condition of magnetic luminosity domination is

$$
P>1.6 \text { s } \dot{P}_{-11}^{1 / 3} I_{45}^{1 / 3} L_{B, 35}^{-1 / 3}(B) .
$$

It is found that for the typical values of $P$ and $\dot{P}$ of magnetars, these objects all lie in the magnetic-dominated regime. Nonetheless, they are not far from the transition boundary. More important, all magnetars ought to be born with millisecond initial period to ensure vigorous dynamo process to occur (Thompson \& Duncan 1993), which means that over the early lifetime of a magnetar, the spindown energy should be the dominant energy source. Even at the present epoch (for typical $P$ and $\dot{P}$ of magnetars), the spindown luminosities (which marks the magnitudes of the pulsar behaviors) are not too low. In fact, many pulsars with such similar $L_{s d}$ 's are detected to be active.

Then there comes a missing link between the magnetars and the radio pulsars. These two types of isolated neutron stars seem to solely manifest the two types of energy sources, respectively. The spindown energy is clearly manifested in pulsars in terms of coherent radio emission, and non-thermal gamma-ray and X-ray emission; while in magnetars the magnetic dissipation energy is manifested in the form of luminous Xrays in the quiescent state and of soft gamma-rays in the burst state. Within the dominant energy output channel for the spindown luminosity, i.e., the radio band and the gamma-ray band, magnetars are not firmly detected. If lack of magnetic-dominated behavior in normal pulsars is understandable because of their weak fields involved, non-detection of 
the spindown-powered behavior in magnetars is in principle not justified. It is worth emphasizing that lack of radio and gamma-ray emission is the prediction of the accretion model for AXPs. Therefore studying the spindown-powered behavior from magnetars is of great theoretical and observational interests. Only when any spindown-powered behavior is firmly detected in SGRs/AXPs, could the accretion model be completely ruled out, and hence, presenting a final proof of the magnetar interpretation.

\section{Spindown-powered activity in magnetars}

The pulsar behavior is marked by the pair-production activity in the magnetosphere. Particles are believed to be accelerated in gaps either in the polar cap region near the surface (Ruderman \& Sutherland 1975; Arons \& Scharlemann 1979; Harding \& Muslimov 1998) or above the null charge surface (Cheng, Ho \& Ruderman 1986). Accelerated primary particles radiate through curvature radiation or inverse Compton scattering, and the resultant gamma-rays produce electron-positron pairs either through one photon $\left(\gamma(B) \rightarrow e^{+} e^{-}(B)\right)$ or two photon $\left(\gamma \gamma \rightarrow e^{+} e^{-}\right)$ processes. In the polar cap region, the secondary pairs also radiate via synchrotron radiation and inverse Compton scattering, leading to a photon-pair cascade (Daugherty \& Harding 1996; Zhang \& Harding 2000a). The condition that pair production is prohibited defines radio pulsar death. Conventionally, this is defined through an energy budget criterion that requires a minimum potential to accelerate particles to a high enough energy in order to allow pair production to occur. This defines a pulsar death valley in the long $P$ regime (e.g. Zhang $2002 \mathrm{~b}$ for a review). According to this criterion, the known magnetars are well above the death line, so that their spindown-powered activity is in principle not prohibited.

In order to interpret the apparent radio quiescence of SGRs/AXPs, Baring \& Harding $(1998,2001)$ argued that pair production is suppressed in magnetars by another more exotic QED process, i.e., magnetic photon splitting. This interpretation relies on the assumption that all three photon splitting modes permitted by charge-parity invariance operate together due to (possible) strong vacuum dispersion effect in superstrong magnetic fields, so that photons with both $\perp$ and $\|$ polarization modes can split. In such a case, for a high enough magnetic field strength, photon splitting will overwhelm magnetic one photon pair production, so that gamma-rays essentially split to photons with lower energies before being materialized, and the magnetar magnetosphere is essentially pair free. Zhang (2001) later found that even if one photon pair pro- 
duction can be completely suppressed by photon splitting (as conjectured by Baring \& Harding), pairs may be formed via two-photon pair production, essentially because the magnetar near surface region is a hot environment with a copious soft photon bath generated from magnetic dissipation. Another issue is that, as long as particles can keep being accelerated to higher altitudes where magnetic field strength is considerably degraded, one photon pair production will overtake photon splitting. This operates for the case of a inner gap type invoking spacecharge-limited flow (Zhang \& Harding 2000b). Both arguments suggest that a magnetar magnetosphere may not be pair free.

Now that the magnetar magnetospheric activity does not differ from that of radio pulsars intrinsically, there are good reasons to expect pulsar-like spin-powered activities from magnetars.

1. Low frequency coherent emission from magnetars? If pairs are not prohibited in the magnetar magnetosphere, why SGRs/AXPs are silent in the conventional radio band? There could be several possible reasons. The most straightforward possibility is that they are actually radio loud, but the radio beams do not sweep towards us due to a very narrow beaming angle of a slow rotator (Gaensler et al. 2001). Other possibilities include that the typical coherent emission frequency is not in the conventional radio band (Zhang 2001; Eichler, Gedalin \& Lyubarsky 2002), or that the coherent condition is fragile and is destroyed in the hot and twisted magnetospheric environment.

2. Non-thermal high energy emission from magnetars? Nonthermal high energy emission is expected from both polar cap cascades and/or from outer gaps in magnetars. In the outer gap scenario, the gamma-ray luminosities of the magnetars have been recently predicted (Cheng \& Zhang 2001; Zhang \& Cheng 2002), which are consistent with the current upper limits on these objects. According to these predictions, some SGRs/AXPs should be detectable by the next generation gammaray detector, GLAST. In the polar cap scenario, high energy emission is also expected, but the typical spectrum would be considerbly shifted to the softer regime due to the large opacities of the gamma-rays (due to one-photon, two-photon pair production and photon splitting). Also the beaming angle is correspondingly smaller. More work in this direction needs to be carried out.

3. High energy neutrinos from magnetars? Zhang, Dai, Mészáros \& Waxman (2002) recently discussed another possible consequence of the magnetar spindown-powered activity. The discussion is relevant to one half of the magnetar population, i.e., those with favorable geometry such that positive ions (likely protons or light nuclei) are accelerated from the polar cap region. For those magnetars that rotate rapidly enough, 
the acceleration potential would be enough to accelerate protons to the energies above the photonmeson threshold, so that these protons will interact with the soft photon bath near the surface and produce pions and neutrinos. The condition for the photomeson interaction threshold is $P<(2.4-6.8)$ s $B_{p, 15}^{1 / 2} R_{6}^{3 / 2}$. This defines a "neutrino death valley" in the magnetar $P-\dot{P}$ (or $P-B_{p}$ ) space. Four magnetars are found to be within or slightly below the valley, which means that under favorable conditions, they are high energy neutrino emitters. Taking into account pion cooling, the typical neutrino energy is several TeV. For on-beam detections, SGR 1900+14 and 1E 1048-5937 have substantial neutrino fluxes, making them interesting targets for the planned large area Cherenkov detectors. The whole magnetar population in the universe adds an interesting contribution to the diffuse high energy neutrino background and the diffuse gamma-ray background.

In the above discussions about magnetar gap accelerations, a dipole configuration is assumed, whilst a magnetar magnetosphere is certainly non-dipole. More specifically, Thompson et al. (2002) argue that the SGR/AXP phenomenology is consistent with the hypothesis that the magnetar magnetosphere is globally twisted. It would be interesting to study the charge-depleted acceleration regions in such a twisted magnetosphere, both near the polar cap region and in the "outer gap" region. A careful study in this direction is called for.

\section{Concluding remark}

Current data reveals a missing link between magnetars and pulsars. Several theoretical efforts have been made to predict spindown-powered activities in magnetars. Connecting this missing link with future observations would provide a solid proof that SGRs/AXPs are indeed isolated neutron stars with strong magnetic fields, i.e., magnetars.

\section{Acknowledgment}

I thank A. K. Harding, P. Mészáros, Z. G. Dai, E. Waxman, R. X. Xu and G. J. Qiao for stimulating collaborations on various topics covered in this review. My research at Penn State University has been supported by NASA grants NAG 5-9192 and NAG 5-9153.

\section{Notes}

1. The internal compositions and equations-of-state of "neutron stars" are not well determined. These stars could be in principle more exotic, e.g., could be composed of pure strange quark matter (e.g. Xu 2002, in these proceedings). Here I refer to "neutron stars" as a broader class of objects that includes more exotic categories. 


\section{References}

Alcock, C., Farhi, E., \& Olinto, A. 1986, Phys. Rev. Lett. 57, 2088

Alpar, M. A. 2001, ApJ, 554, 1245

Arons, J., \& Scharlemann, E.T. 1979, ApJ, 231, 854

Baring, M. G., \& Harding, A. K. 1998, ApJ, 507, L55

Baring, M. G., \& Harding, A. K. 2001, ApJ, 547, 929

Chatterjee, P., Herquist, L. \& Narayan, R. 2000, ApJ, 534, 373

Cheng, K. S. \& Dai, Z. G. 1998, Phys. Rev. Lett., 80, 18

Cheng, K. S., Ho, C. \& Ruderman, M.A. 1986, ApJ, 300, 500

Cheng, K. S. \& Zhang, L. 2001, ApJ, 562, 918

Daugherty, J.K., \& Harding, A.K. 1996, ApJ, 458, 278

Duncan, R. C. \& Thompson, C. 1992, ApJ, 392, L9

Eichler, D., Gedalin, M. \& Lyubarsky, Y. 2002, ApJ, 578, L121

Gaensler, B. M., Slane, P. O., Gotthelf, E. V. \& Vasisht, G. 2001, ApJ, 559, 963

Gavriil, F. P., Kaspi, V. M., \& Woods, P. M. 2002, Nature, 419, 142

Harding, A. K., \& Muslimov, A.G. 1998, ApJ, 508, 328

Heyl, J. S. \& Hernquist, L. 1997, ApJ, 489, L67

Ibrahim, A. I. et al. 2002, 574, L51

Kaspi, V. M. \& Gavriil, F. P. 2002, IAUC 7924

Kulkarni, S. R., Kaplan, D. L., et al. 2003, ApJ, in press (astro-ph/0209520)

Marsden, D. et al. 2001, ApJ, 550, 397

Paczyński, B. 1990, ApJ, 365, L9

Paczyński, B. 1992, Acta Astronomica, 42, 145

Pavlov, G. G., Zavlin, V. E. \& Sanwal, D. 2002, astro-ph/0206024

Ruderman, M.A., \& Sutherland, P.G. 1975, ApJ, 196, 51

Thompson, C. \& Duncan, R. C. 1993, ApJ, 408, 194

Thompson, C. \& Duncan, R. C. 1995, MNRAS, 275, 255

Thompson, C. \& Duncan, R. C. 1996, ApJ, 473, 322

Thompson, C., Lyutikov, M. \& Kulkarni, S. R. 2002, ApJ, 574, 332

Usov, V. V. 1993, ApJ, 410, 761

Usov, V. V. 2001, Phys. Rev. Lett., 87(2), 021101

$\mathrm{Xu}, \mathrm{R}$. X. 2002, in these proceedings (astro-ph/0211563)

Zhang, B. 2001, ApJ, 562, L59

Zhang, B. 2002a, Mem. S. A. It., 73 (2), 516 (astro-ph/0102098)

Zhang, B. 2002b, In: J.L. Han \& R. Wielebinski (eds) Proc. of Sino-German Radio Astronomy Conference on Radio Studies of Galactic Objects, Galaxies and AGNs, special issue of ChA\&A (astro-ph/0209160)

Zhang, B., Dai, Z. G., Mészáros, P. \& Waxman, E. 2002, Phys. Rev. Lett., submitted (astro-ph/0210382)

Zhang, B. \& Harding, A. K. 2000a, ApJ, 532, 1150

Zhang, B. \& Harding, A. K. 2000b, ApJ, 535, L51

Zhang, B., Xu, R. X. \& Qiao, G. J. 2000, ApJ, 545, L127

Zhang, L. \& Cheng, K. S. 2002, ApJ, 579, 716 